Proceedings of the LIV Zakopane School of Physics, Breaking Frontiers, Zakopane, Poland, May 21-25, 2019

\title{
Magnetic Anisotropy and Temperature Dependence of Exchange Bias in Epitaxial $\mathrm{CoO}(111) / \mathrm{Fe}(110)$ Bilayers
}

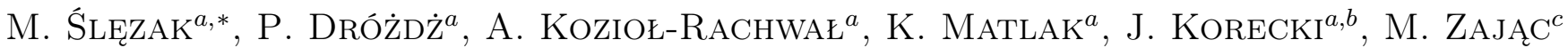 \\ AND T. ŚLĘZAK ${ }^{a}$ \\ ${ }^{a}$ AGH University of Science and Technology, Faculty of Physics and Applied Computer Science, \\ 30-059 Kraków, Poland \\ ${ }^{b}$ Jerzy Haber Institute of Catalysis and Surface Chemistry, Polish Academy of Sciences,30-239 Kraków, Poland \\ ${ }^{c}$ National Synchrotron Radiation Centre SOLARIS, Jagiellonian University, Kraków, Poland
}

\begin{abstract}
We report on the in-plane magnetic anisotropy and temperature dependence of the exchange bias in epitaxial $\mathrm{CoO}(111) / \mathrm{Fe}(110)$ bilayers. The Fe thickness induced in-plane spin reorientation transition and the existence of the exchange bias below the blocking temperature $\approx 27 \mathrm{~K}$ is documented in $\mathrm{CoO}(111) / \mathrm{Fe}(110)$ system. For $d_{F e}=80 \AA$, we find that the hysteresis loop shift-field, $H_{\mathrm{EB}}$, reaches the value $\approx 250$ Oe at $184 \mathrm{~K}$. A true interplay between antiferromagnetic $\mathrm{CoO}$ and ferromagnetic Fe layers is described.
\end{abstract}

DOI: 10.12693/APhysPolA.137.44

PACS/topics: magnetic anisotropy, exchange bias, thin films

\section{Introduction}

Exchange coupled ferromagnetic/antiferromagnetic (FM/AFM) bilayers [1, 2] have been intensively investigated mainly due to their technological applications in spin valves and magnetic tunnel junctions. In FM/AFM systems a particularly interesting and fundamentally important effect, namely the exchange-bias (EB) [3, 4] at the FM/AFM boundary, is manifested by the shift of the hysteresis loop and an increase of its coercivity. The magnetic properties of the exchange bias systems depend on the anisotropies of both the ferromagnet and the antiferromagnet. For this reason systematic experimental studies on systems, where strong and well defined FM anisotropy and exchange bias interaction coexist and interplay, are important.

We studied $\mathrm{CoO} / \mathrm{Fe}(110)$ bilayers epitaxially grown on a W(110) single crystal substrate. In $\mathrm{Fe}(110)$ films on $\mathrm{W}(110)$, the magnetization rotates from the $[1-10]$ to [001] in-plane direction above a critical thickness of spin reorientation transition (SRT) [5-7]. Such thicknessinduced in-plane SRT was shown to occur in variety of bilayers, such as $\mathrm{Au} / \mathrm{Fe}(110), \mathrm{Ag} / \mathrm{Fe}(110)[8,9]$ or $\mathrm{Co} / \mathrm{Fe}(110)[10,11]$ and $\mathrm{Au} / \mathrm{Co} / \mathrm{Fe}(110)$ [12]. Here we report on SRT and exchange bias in $\mathrm{Fe}(110) / \mathrm{W}(110)$ films coated with a metal-oxide layer (i.e., AFM CoO). For this system, we have recently shown how a ferromagnetic Fe layer can be used to tailor the magnetic anisotropy of the antiferromagnetic $\mathrm{CoO}$ overlayer [13]. In the present report we extend this study and present temperature dependence of exchange bias in $\mathrm{CoO}(111) / \mathrm{Fe}(110)$ bilayers. We complement conclusions described in Ref. [13] by showing a true bilateral nature

\footnotetext{
* corresponding author
}

of FM-AFM interaction. Specifically we show that depending on the orientation of the frozen $\mathrm{CoO}$ spins, that can be controlled either by remanent magnetic state of the $\mathrm{Fe}$ or by applied field cool procedure, it is possible to strongly modify the magnetic anisotropy field of the $\mathrm{Fe}(110)$ layer.

\section{Experimental and results}

The W(110) crystal cleaning procedure [14], applied in ultra-high vacuum (UHV) chamber, consisted of repeated annealing cycles in an oxygen atmosphere $\left(5 \times 10^{-8}\right.$ mbar at $1200^{\circ} \mathrm{C}$ ) and subsequent flash heating to $1800^{\circ} \mathrm{C}$. We used molecular beam epitaxy (MBE) technique to grow wedged $\mathrm{Fe}(110)$ films on $\mathrm{W}(110)$ at room temperature. After the preparation, the Fe films with thickness $d_{\mathrm{Fe}}$ ranging from 80 to $300 \AA$, were post-annealed at $675 \mathrm{~K}$. Next, a $90 \AA$ thick $\mathrm{CoO}$ adlayer was grown on the $\mathrm{Fe}(110)$ wedge-films by reactive deposition of cobalt at a temperature of $470 \mathrm{~K}$ and at an $\mathrm{O}_{2}$ partial pressure of $5 \times 10^{-7} \mathrm{mbar}$. The low-energy electron diffraction (LEED) pattern from the surface of the CoO-covered $\mathrm{Fe}(110) / \mathrm{W}(110)$ sample indicated a hexagonal $\mathrm{CoO}(111)$ surface structure throughout the underlying Fe film. The X-ray absorption spectroscopy (XAS) spectra, ex situ measured at the XAS end station of National Synchrotron Radiation Centre Solaris in Kraków, indicate a proper stoichiometry of such prepared CoO overlayer, as deduced from comparison of the measured XAS intensity peak structure with literature data [15], see Fig. 1.

The magnetic properties of the $\mathrm{CoO} / \mathrm{Fe}(110)$ system were studied in situ, using the longitudinal magnetooptic Kerr effect (MOKE). In Fig. 2, we present magnetic hysteresis loops acquired at $T=300 \mathrm{~K}$ with MOKE, for selected Fe thicknesses: $d_{\mathrm{Fe}}=80,120,150,160$, $170,200,250$, and $300 \AA$. Up to $d_{\mathrm{Fe}} \approx 150 \AA$, square 


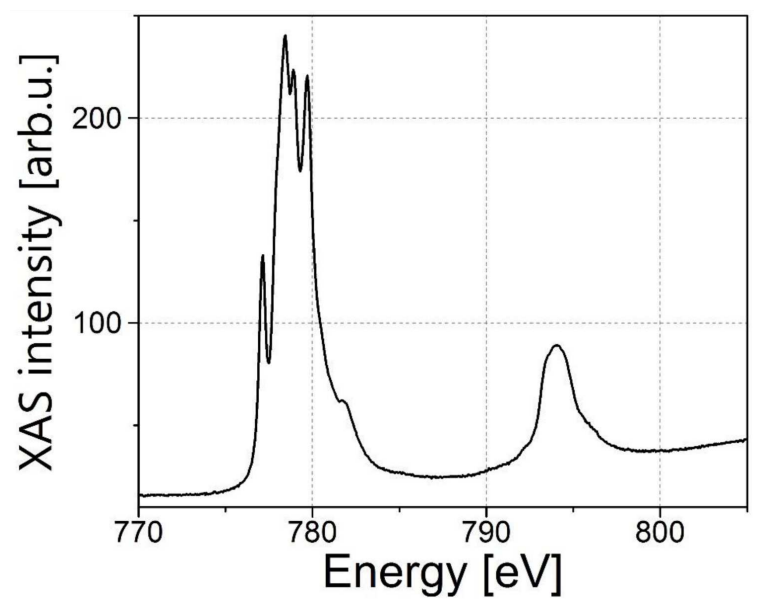

Fig. 1. The XAS spectra of $\mathrm{CoO}(111) / \mathrm{Fe}(110)$ bilayer in the energy range that covers the $L_{3}$ and $L_{2}$ absorption edges of Co.

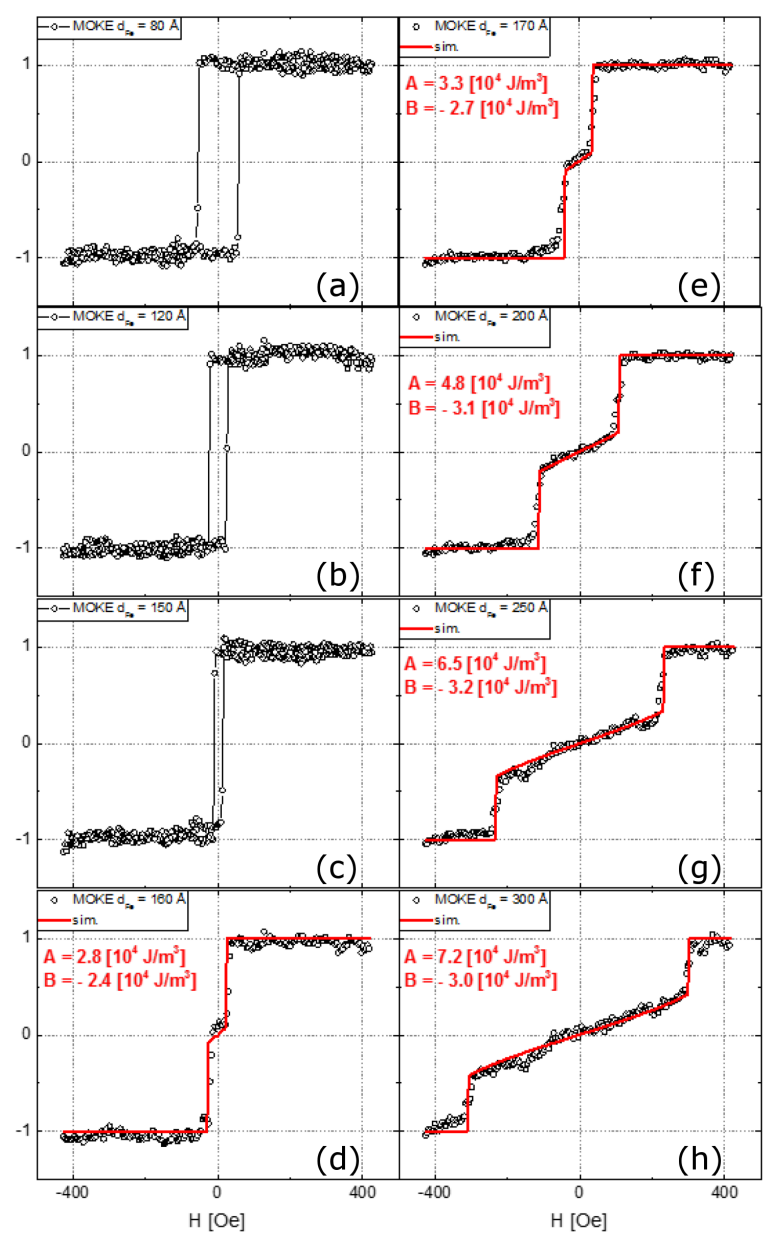

Fig. 2. Room temperature magnetic hysteresis loops measured using MOKE (black open dots) for $d_{\mathrm{Fe}}=80-300 \AA$. Starting from $d_{\mathrm{Fe}}=160 \AA$ (d) hard axis hysteresis loops are compared with simulations (red solid lines). The external magnetic field $H$ was applied along the $[1-10]$ direction in the $\mathrm{Fe}(110)$ plane. Magnetic anisotropy constants, $A$ and $B$, used for each particular simulation are given in corresponding insets. hysteresis loops, typical for the easy magnetization direction are observed (Fig. $2 \mathrm{a}-\mathrm{c}$ ), which means that for thin $\mathrm{Fe},[1-10]$ is the easy direction of magnetization in $\mathrm{CoO}(111) / \mathrm{Fe}(110)$ bilayers. Starting from $d_{\mathrm{Fe}} \approx 160 \AA$ the magnetic hysteresis loops become characteristic for the hard magnetization axis (Fig. $2 \mathrm{~d}-\mathrm{h}$ ), which indicates that the $\mathrm{CoO} / \mathrm{Fe}$ bilayer passed the Fe thickness induced in-plane SRT. The hard axis loops are almost hysteresisless, therefore in Fig. $2 \mathrm{~d}-\mathrm{h}$ only one branch of the measured magnetization curves is shown for clarity.

In order to determine the in-plane magnetic anisotropy (MA) constants for $\mathrm{CoO}(111) / \mathrm{Fe}(110)$ bilayer, we performed simulations of the hard axis magnetic hysteresis loops for the 160-300 $\AA$ range of $d_{\mathrm{Fe}}$. We describe the magnetization reversal within the coherent rotation model. Simulated magnetic hysteresis loops are obtained from the minimization of the free enthalpy density, $G$, as a function of the external magnetic field, $H$ :

$$
\begin{aligned}
G & =-\frac{K_{\mathrm{EB}}}{d_{\mathrm{Fe}} \cos (\Phi)}+A \cos ^{2}(\Phi)+B \cos ^{4}(\Phi) \\
& -M_{s} H \cos (\Phi),
\end{aligned}
$$

where $M_{s}$ is the saturation magnetization (its value can be assumed to be bulk-like for such thick Fe films), $\Phi$ defines the orientation of the Fe magnetization with respect to the $\mathrm{Fe}[1-10]$ in-plane direction, and $K_{\mathrm{EB}}$ is the $\mathrm{CoO} / \mathrm{Fe}$ exchange-coupling constant. $A$ and $B$ are the second- and fourth-order effective MA constants, respectively. They can be defined in terms of the volume and surface MA contributions:

$$
\begin{aligned}
& A=K_{v, p} K_{s, p} / d_{\mathrm{Fe}}, \\
& B=K_{v, p p} K_{s, p p} / d_{\mathrm{Fe}} .
\end{aligned}
$$

In Eqs. (2) and (3), $K_{v, p}$ and $K_{v, p p}$ are the second- and fourth-order volume constants of the in-plane MA, while $K_{s, p}$ and $K_{s, p p}$ denote their surface analogues [9]. Since all RT loops are practically hysteresis-less, in all simulated hysteresis loops presented in Fig. $2 \mathrm{~d}-\mathrm{h}$ (red solid lines) a global minimum of energy was found for each value of external magnetic field. At $\mathrm{RT}$ there is no EB interaction in the $\mathrm{CoO} / \mathrm{Fe}$ bilayers, therefore $K_{\mathrm{EB}}$ value was kept zero in all corresponding simulations. For each $d_{\mathrm{Fe}}$, the values of $A$ and $B$ were tuned to obtain the best match between the simulated and experimental hysteresis curves. The dependence of $A$ and $B$ on the inverse of the Fe thickness $\left(1 / d_{\mathrm{Fe}}\right)$ is plotted in Fig. 3.

Clearly, $A$ and $B$ constants follow a linear dependence on $1 / d_{\mathrm{Fe}}$. From linear regression fits, as presented in Fig. 3, we find $K_{v, p}=12.5 \times 10^{4} \mathrm{~J} / \mathrm{m}^{3}$, $K_{s, p}=1.5 \mathrm{~mJ} / \mathrm{m}^{2}, K_{v, p p}=-3.6 \times 10^{4} \mathrm{~J} / \mathrm{m}^{3}$, and $K_{s, p p}=-0.1 \mathrm{~mJ} / \mathrm{m}^{2}$. The $K_{v, p}$ and $K_{s, p}$ values determined here for $\mathrm{CoO}(111) / \mathrm{Fe}(110)$ bilayers can be compared to their analogues that we have recently reported for uncovered $\mathrm{Fe}(110)$ grown in the same UHV system and using identical preparation conditions [10]. The volume contribution $K_{v, p}$ is significantly enhanced by $\mathrm{CoO}$ capping as compared to uncovered Fe(110) films 


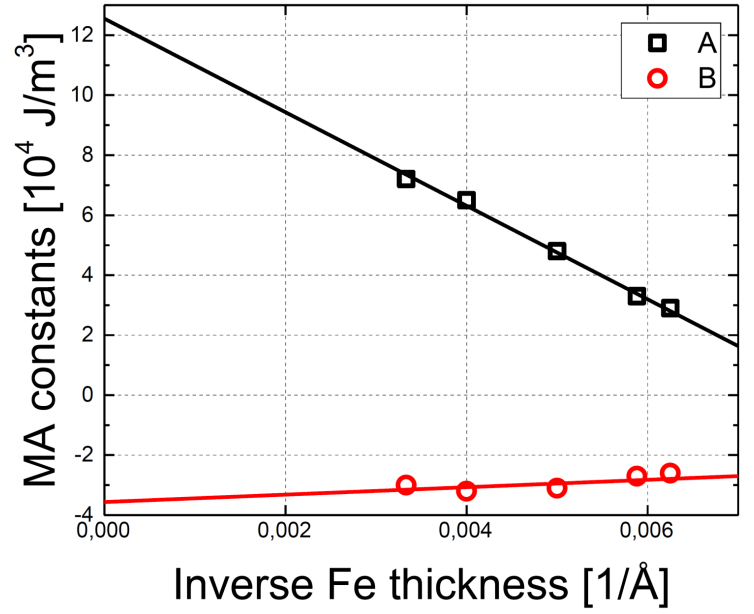

Fig. 3. Inverse-thickness, $1 / d_{\mathrm{Fe}}$, dependence of the second- and fourth-order effective MA constants, $A$ and $B$, as determined from magnetic hysteresis loops simulations. Lines represent linear regression fits from which surface and volume contributions to $A$ and $B$ are determined, see text for details.

$\left(K v, p=10.60 \times 10^{4} \mathrm{~J} / \mathrm{m}^{3}[10]\right)$ and this can be interpreted in terms of additional epitaxial strain introduced at the $\mathrm{CoO} / \mathrm{Fe}$ interface. Even more drastic change is observed for the surface contribution, which for uncovered $\mathrm{Fe}(110)$ is almost twice smaller $\left(0.8 \mathrm{~mJ} / \mathrm{m}^{2}[10]\right)$ when compared to the present $\mathrm{CoO} / \mathrm{Fe}$ study. To conclude this part, contrary to a decrease of the magnetic surface anisotropy (MSA) usually observed in the case of noble metal $(\mathrm{Ag}, \mathrm{Au})$ adlayers $[9,10]$ or adsorption of oxygen [9] or UHV residual gases, $\mathrm{Co}[10]$ and $\mathrm{CoO}$ (present report) are the capping layers that can be used to efficiently increase the in-plane MSA at the $\mathrm{Fe}(110)$ surface.

The temperature dependence of exchange bias, described here by the shift field of the magnetic hysteresis loop $H_{\mathrm{EB}}(T)$, is presented in Fig. 4a for $d_{\mathrm{Fe}}=80 \AA$. A close to linear dependence of $H_{\mathrm{EB}}$ on temperature is observed and the blocking temperature of $\mathrm{CoO}(111)$ on $\mathrm{Fe}(110)$ can be estimated as $T_{B} \approx 270 \mathrm{~K}$. It should be noted that in our system exchange bias is present even without field-cool (FC) procedure, however, particular choice of magnetic field orientation during FC is crucial for the orientation of AFM frozen spins and consequently for the magnitude of the shift field $H_{\mathrm{EB}}$. When the sample is cooled at remnant magnetic state, we find that the orientation of AFM frozen spins follows the local (thickness dependent) orientation of Fe magnetization, in agreement with our previous report [13]. In case of FC procedure, when FC magnetic field is parallel to the local easy axis of $\mathrm{Fe}$ layer, the corresponding value of $H_{\mathrm{EB}}$ is the same like for the sample cooled at its remnant magnetic state. On the other hand, when the sample is cooled in magnetic field oriented along Fe in-plane hard axis and high enough to magnetically saturate the $\mathrm{Fe}$ layer, the orientation of AFM spins switches to direction
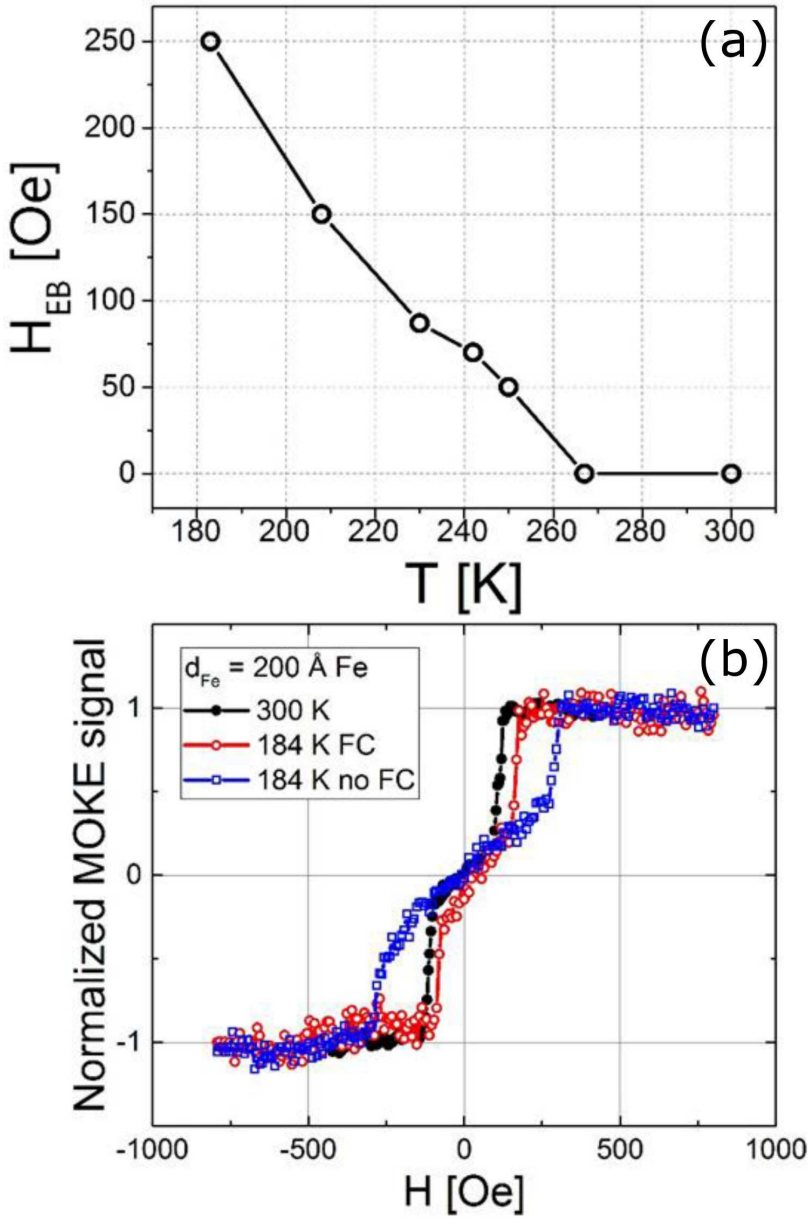

Fig. 4. (a) The temperature dependence of the magnetic hysteresis loop shift field $H_{\mathrm{EB}}$ as determined for $d_{\mathrm{Fe}}=80 \AA$, for sample cooled at its remnant magnetic state. The solid line serves as a guide for the eye. (b) Magnetic hysteresis loops measured using MOKE for $d_{\mathrm{Fe}}=200 \AA$. The sample temperature is $T=300 \mathrm{~K}$ (full symbols) and $184 \mathrm{~K}$ for the sample field-cooled (FC, open circles) and cooled at remnant magnetic state (denoted as no FC, open squares). The external magnetic field $\mathrm{H}$ was applied along the $[1-10]$ direction in the Fe(110) plane, for both measurements and field-cool procedure.

parallel to the FM hard axis. In such a case the shift field increases when the orientation of AFM spins is parallel to the direction of magnetic field applied during magnetization reversal.

We also observe the impact of AFM unidirectional magnetic anisotropy on the uniaxial magnetic anisotropy of FM layer. For $d_{\mathrm{Fe}}=200 \AA$, when the FC procedure is applied, the anisotropy field of the typical hard axis hysteresis loop changes very slightly with decreasing temperature, compare hysteresis curves at $300 \mathrm{~K}$ and at $184 \mathrm{~K}$ (FC) in Fig. 4b. This is because $\mathrm{CoO}$ spins are frozen along $\mathrm{Fe}[1-10]$ direction and do not support the intrinsic, [001] magnetic anisotropy of $200 \AA$ thick Fe layer. Situation changes drastically when the sample is cooled 
to $184 \mathrm{~K}$ at the remnant magnetic state of Fe. In this case, Fe which is magnetized along [001] direction freezes the $\mathrm{CoO}$ spins along $\mathrm{Fe}[001]$ direction which results in a strong enhancement of magnetic anisotropy field, see hysteresis curve marked with open squares in Fig. $4 \mathrm{~b}$.

\section{Conclusion}

We have studied the $\mathrm{CoO}(111) / \mathrm{Fe}(110)$ bilayer epitaxial system and we report on its in-plane MA and temperature dependence of exchange bias interaction. The Fe thickness induced in plane SRT observed in the $\mathrm{Fe}(110)$ films covered with $\mathrm{CoO}$ has its critical thickness $d_{\mathrm{Fe}}=160 \pm 5 \AA$ depending on the temperature. The blocking temperature for $90 \AA$ thick $\mathrm{CoO}(111)$ adlayer is found to be close to the bulk value of $\mathrm{CoO}$ Néel temperature. A true interplay between AFM ( $\mathrm{CoO})$ and FM (Fe) layers is observed, namely the orientation FM magnetization determines the easy axis of AFM layer, but on the other hand AFM frozen spins can be used to strongly increase the in-plane uniaxial magnetic anisotropy of the neighboring FM.

\section{Acknowledgments}

This work is supported by the AGH University of Science and Technology statutory task No. 11.11.220.01/6 within subsidy of the Ministry of Science and Higher Education. A.K.-R. was supported by the "Antiferromagnetic proximity effect and development of epitaxial bimetallic antiferromagnets - two routes towards next-generation spintronics" project which is carried out within the Homing programme of the Foundation for Polish Science co-financed by the European Union under the European Regional Development Fund.

The authors acknowledge the CERIC-ERIC Consortium for financial support through the research grant MAG-ALCHEMI.

\section{References}

[1] R. Morales, A.C. Basaran, J.E. Villegas, D. Navas, N. Soriano, B. Mora, C. Redondo, X. Batlle, I.K. Schuller, Phys. Rev. Lett. 114, 097202 (2015).

[2] C. Leighton, M.R. Fitzsimmons, P. Yashar, A. Hoffmann, J. Nogues, J. Dura, C.F. Majkrzak, I.K. Schuller, Phys. Rev. Lett. 86, 4394 (2001).
[3] W.H. Meiklejohn, C.P. Bean, Phys. Rev. 105, 904 (1957).

[4] P.K. Manna, S.M. Yusuf, Phys. Rep. 535, 61 (2014).

[5] U. Gradmann, J. Korecki, G. Waller, Appl. Phys. A 39, 101 (1986).

[6] T. Ślęzak, M. Zaja,c, M. Ślęzak, K. Matlak, A. Kozioł-Rachwał, D. Wilgocka-Ślęzak, A.I. Chumakov, R. Rüffer, J. Korecki, Phys. Rev. B 87, 094423 (2013).

[7] M. Ślęzak, T. Giela, D. Wilgocka-Ślęzak, A. KoziołRachwał, T. Ślęzak, R. Zdyb, N. Spiridis, C. Quitmann, J. Raabe, N. Pilet, J. Korecki, J. Magn. Magn. Mater. 348, 101 (2013).

[8] I.G. Baek, H. Lee, H.J. Kim, E. Vescovo, Phys. Rev. B 67, 075401 (2003).

[9] H.J. Elmers, U. Gradmann, Appl. Phys. A 51, 255 (1990).

[10] M. Ślęzak, T. Ślęzak, K. Matlak, B. Matlak, P. Dróżdż, T. Giela, D. Wilgocka-Ślęzak, N. Pilet, J. Raabe, A. Kozioł-Rachwał, J. Korecki, Phys. Rev. B 94, 014402 (2016).

[11] M. Ślęzak, T. Ślęzak, K. Matlak, P. Drózdz, J. Korecki, AIP Adv. 8, 056806 (2018).

[12] M. Ślęzak, P. Dróżdż, K. Matlak, A. Kozioł-Rachwał, J. Korecki, T. Ślęzak, J. Magn. Magn. Mater. 475, 195 (2019).

[13] M. Ślęzak, T. Ślęzak, P. Dróżdż, B. Matlak, K. Matlak, A. Kozioł-Rachwał, M. Zając, J. Korecki, Sci. Rep. 9, 889 (2019).

[14] K. Freindl, E. Partyka-Jankowska, W. Karaś, M. Zaja, c, E. Madej, N. Spiridis, M. Ślęzak, T. Ślęzak, D. Wiśnios, J. Korecki, Surf. Sci. 617, 183 (2013).

[15] J. Wu, J.S. Park, W. Kim, E. Arenholz, M. Liberati, A. Scholl, Y.Z. Wu, C. Hwang, Z.Q. Qiu, Phys. Rev. Lett. 104, 217204 (2010). 